\section{A) Check for updates}

Cite this: Food Funct., 2020, 11, 617

\title{
Effect of semolina pudding prepared from starch branching enzyme Ila and $b$ mutant wheat on glycaemic response in vitro and in vivo: a randomised controlled pilot study $\dagger$
}

\author{
Marina Corrado, (DD a Anna Cherta-Murillo, (D) ${ }^{b}$ Edward S. Chambers, ${ }^{b}$ \\ Abigail J. Wood, ${ }^{c}$ Amy Plummer, ${ }^{c}$ Alison Lovegrove, (D) ${ }^{c}$ Cathrina H. Edwards, (D) ${ }^{a}$ \\ Gary S. Frost ${ }^{b}$ and Brittany A. Hazard (D)*a,d
}

\begin{abstract}
Refined starchy foods are usually rapidly digested, leading to poor glycaemic control, but not all starchy foods are the same. Complex carbohydrates like resistant starch (RS) have been shown to reduce the metabolic risk factors for chronic diseases such as hyperglycaemia and overweight. The aim of the project was to develop a semolina-based food made from a starch branching enzyme // (sbella/b-AB) durum wheat mutant with a high RS content and to measure its glycaemic index using a double-blind randomised pilot study. We report here the amylose, RS and non-starch polysaccharide concentration of raw sbella/b-AB and wild-type control (WT) semolina. We measured RS after cooking to identify a model food for in vivo testing. Retrograded sbella/b-AB semolina showed a higher RS concentration than the WT control (RS $=4.87 \pm 0.6 \mathrm{~g}$ per $100 \mathrm{~g}, 0.77 \pm 0.34 \mathrm{~g}$ per $100 \mathrm{~g}$ starch DWB, respectively), so pudding was selected as the test food. Ten healthy participants consumed $\sim 50 \mathrm{~g}$ of total starch from WT and sbella/b-AB pudding and a standard glucose drink. Capillary blood glucose concentrations were measured in the fasting and postprandial state $(2 \mathrm{~h})$ : incremental area-under-the-curve (iAUC) and $\mathrm{Gl}$ were calculated. We found no evidence of difference in Gl between sbella/b-AB pudding and the WT control, but the starch digestibility was significantly lower in sbella/b-AB pudding compared to the WT control in vitro $\left(C_{90}=33.29 \%\right.$ and $47.38 \%$, respectively). Based on these results, novel sbella/b-AB wheat foods will be used in future in vivo studies to test the effect of different RS concentrations and different food matrices on glycaemia.
\end{abstract}

Received 21st October 2019, Accepted 9th December 2019 DOI: $10.1039 /$ c9fo02460c rsc.li/food-function sharp increase in the postprandial blood glucose concentration and are therefore described as having a high glycaemic index. ${ }^{4}$ Starch that is digested more slowly is expected to result in a more gradual postprandial glycaemic response. In some cases, starch can resist or escape digestion in the small intestine ('resistant starch'), moving into the large intestine where it may be fermented by the resident microbiota into shortchain fatty acids (SCFAs). This is believed to have positive effects on glucose metabolism. ${ }^{5,6}$

Starch is a ubiquitous storage polysaccharide in wheat endosperm and the main source of digestible carbohydrates; however, its digestibility depends on its native structure in the grain and physical form in foods. In the native state, starch exists as dense granules where the $\alpha$-glucan chains are not readily available for amylase digestion. ${ }^{7,8}$ Most starch-based foods are cooked to improve digestibility as well as texture; heating starch in excess water results in water absorption and swelling of the starch granules leading to loss of native resistant starch (RS), a process known as gelatinisation. As the starch cools, starch polysaccharides tend to re-associate in a 
more ordered and crystalline state (retrogradation) becoming more resistant to digestion. ${ }^{9}$ The physicochemical modifications that starch undergoes during processing, together with the native starch structure, can alter the resistance of starch to digestion in the small intestine. ${ }^{10,11}$ In high amylose starches, molecular interactions between amylose-amylose, amyloseamylopectin and amylopectin-amylopectin can lead to retrogradation. During gelatinisation, amylose leaches out from granules as a random coil which tends to form either singlehelical complexes (amylose-lipid complexes) or double helices by self-association. The rate of self-association depends on chain-lengths, concentration and cooling rate; high amylose starches are more likely to form double helical structures which are thought to be resistant to $\alpha$-amylase digestion. ${ }^{12}$ Starch digestibility is also strongly influenced by the surrounding food matrix properties, which can hinder starch gelatinisation during processing conditions and/or limit starch exposure to digestive enzymes and fluids during gastrointestinal transit. $^{13-15}$

Another factor that may influence the digestibility of foods made from wheat semolina is the presence of non-starch polysaccharides (NSPs). ${ }^{16}$ NSPs derived from the grain cell walls completely resist enzymatic digestion in the small intestine. ${ }^{17}$ They are the main source of dietary fibre from durum wheat semolina (1.4 to $1.8 \%$ dry weight), with the major fraction being arabinoxylan $\mathrm{AX}(\sim 70 \%)$ and $(1 \rightarrow 3,1 \rightarrow 4)-\beta$-D-glucan (mixed-linkage $\beta$-glucan or MLG) $(\sim 20 \%) .{ }^{18}$ AX has a backbone of $\beta$-D-xylopyranosyl residues linked by $(1 \rightarrow 4)$ glycosidic linkages with some residues substituted with $\alpha$-L-arabinofuranosyl at positions 2 and/or 3. ${ }^{19,20}$ At high concentrations, AXs, particularly water-soluble AXs, can contribute to an increased meal viscosity and lower starch gelatinisation and hydrolysis, having implications for postprandial glycaemia. ${ }^{21,22}$

In an attempt to increase the fibre content of refined wheat foods and lower their glycaemic index (GI), sbeIIa/b-AB durum wheat was developed by combining mutations in sbeII genes, encoding Starch Branching Enzyme II (SBEII) which catalyzes the addition of branch points during amylopectin biosynthesis. $^{23}$ The partial loss of function of SBEIIa and SBEIIb enzymes during starch formation leads to an increase in the amount of amylose and the proportion of starch that is resistant to digestion in raw semolina, producing pasta of acceptable quality with improved firmness after cooking. ${ }^{24}$ Animal studies have shown a positive fermentation response to sbeIIal $b$-AB wheat provided as pellets with an increase in the production of SCFA and lower colonic $\mathrm{pH}$ in rats fed with sbeIIal $b$-AB flour compared to WT flour for 4 weeks. ${ }^{25,26}$

In pig studies, other forms of RS were found to lower metabolizable energy intake, prolong satiety and decrease the kinetics of glucose absorption while altering gut hormone secretion. $^{27,28}$ In an acute human study, decreased energy intake was observed after consumption of muffins containing high RS compared to a low RS breakfast. ${ }^{29}$ Harrold J. et al. found that consumption of a new composite satiety ingredient made of whole-grain and high-amylose corn flour (30 g of RS on a dry weight basis) for breakfast led to increased satiety and lower energy intake at dinner, compared to the maltodextrin control. ${ }^{30}$

Therefore, increasing resistant starch in wheat seems to be a promising strategy for improving the dietary fibre content of wheat-based foods that could lead to steady glucose release in the gut and a gradual increase in postprandial glucose concentrations. Even though sbeIIa/b-AB wheat is known to have higher amylose and resistant starch concentration than its WT control in the raw state, very little is known about the physiochemical properties of starch and non-starch polysaccharides in sbeIIa/b-AB semolina after cooking and its impact on blood glucose response. In this study, we measured the RS concentration of two model foods, semolina porridge and semolina pudding. Semolina pudding was selected as a model food to measure starch digestibility $\left(C_{90}\right)$ in vitro and to explore pudding GI in vivo, in a double-blind randomised controlled pilot study.

\section{Materials \& methods}

\subsection{Materials}

Durum wheat [T. turgidum L. subsp. Durum (Desf.) Husn.] with high RS and amylose concentration was developed previously at the University of California Davis using the cultivar Kronos by combining mutations in starch branching enzyme II genes $\left(\right.$ sbeIIa/b-AB). ${ }^{31}$ For this study, the sbeIIa/b-AB mutant and WT control plants were grown at the University of California Experimental Field Station in Davis, CA, during the 2014-2015 growing season and harvested in bulk in June 2015.

\subsection{Milling and particle size}

Prior to milling, grains were conditioned using a two-stage water addition and de-branned using a Satake laboratory debranner. Bulked durum wheat grains were milled to obtain semolina using a Bühler laboratory mill at the Allied Milling and Baking Technical Centre (Vanwall Road, Maidenhead, Berkshire, SL6 4UF). The particle size distributions of sbeIIal $b$-AB and WT semolina suspended in water were determined by laser diffraction analysis on a Beckman Coulter LS 13320.

\subsection{Starch extraction from WT and $s b e I I a / b-A B$ mutant grains}

Approximately 50 wheat grains were left overnight at $+4{ }^{\circ} \mathrm{C}$ in a Petri dish on moistened filter paper. They were then ground using a pestle and a mortar in approximately $5 \mathrm{~mL}$ of deionised water, after removing the husk and embryo manually. The extract was then filtered through a layer of Miracloth (475855, Calbiochem $\AA$ ) by washing with $30 \mathrm{~mL}$ of deionised water. The solution was centrifuged, at $2000 \mathrm{~g}$ for $5 \mathrm{~min}$ and the recovered pellet was washed with $30 \mathrm{~mL}$ of $2 \%$ Sodium Dodecyl Sulfate (SDS). The pellet was washed and centrifuged twice in $5 \mathrm{~mL}$ of $2 \%$ SDS. The resulting starch pellet was washed a further three times with $5 \mathrm{~mL}$ of acetone and left to dry overnight in a fume hood to ensure complete evaporation of the solvent. ${ }^{32}$ 


\subsection{Food models: semolina processing for in vitro analyses}

Mutant sbeIIa/b-AB semolina and WT control semolina were gelatinised by adding $1 \mathrm{~mL}$ of deionised water to $\sim 100 \mathrm{mg}$ of semolina followed by boiling for $10 \mathrm{~min}$ at $99{ }^{\circ} \mathrm{C}$ while stirring to ensure homogeneous gelatinisation of the starch (porridge model). The gelatinised samples were cooled on ice overnight with continuous stirring during which some retrogradation occurred (pudding model). The RS concentration of model food products (porridge and pudding) was analysed to identify one food product to be tested in vivo as described below.

\subsection{Formulation and preparation of semolina pudding for in vivo analysis}

Test meals consisted of semolina puddings made from sbeIIa/ $b$-AB or WT control wheat semolina. Both pudding recipes were designed to provide approximately $50 \mathrm{~g}$ of total starch with a constant solid to liquid ratio consisting of $17.02 \%$ semolina, $82.75 \%$ water and $0.23 \%$ salt (w/w fresh weight basis). Dry ingredients (sbeIIa/b-AB or WT semolina and salt) were packed into coded containers at the Quadram Institute Bioscience (QIB) and shipped to the NIHR Imperial Clinical Research Facility, London (UK), where the individual portions were cooked prior to serving such that both participants and researchers preparing the pudding and assessing the outcomes were blinded. Wheat semolina puddings were prepared by simmering at $80^{\circ} \mathrm{C}$ for 3 min while stirring to obtain homogeneous starch gelatinisation and cooled at room temperature for $10 \mathrm{~min}$ before storing at $4^{\circ} \mathrm{C}$ overnight to allow the starch to retrograde. After preliminary analysis of the results, the test meals were unblinded to reveal the treatment and control for completing the statistical analysis.

Each test meal was prepared individually and served with 250 to $400 \mathrm{~mL}$ of water. The volume of water served with the test meal was adjusted to compensate for the water loss during the cooking of the semolina pudding and ensured a constant liquid-solid ratio across all meals.

\subsection{Starch analysis: total starch, amylose, resistant starch and moisture content}

Starch analyses are reported on a dry weight basis. The moisture of unprocessed semolina was determined using a modified version of the AACC (44-15A) air oven method, a one stage procedure, in which the amount of sample used was reduced to $100 \mathrm{mg}$ due to the limited quantity of the available material. The total starch (TS) content of unprocessed semolina was determined using Megazyme's Total Starch Assay Kit $(\mathrm{KOH}$ format, AOAC 996.11). The resistant starch (RS) content was measured using Megazyme's Assay Kit (AOAC 2002.02) following the manufacturer's instructions.

\subsection{Amylose determination}

$0.05 \%(\mathrm{w} / \mathrm{v})$ suspension of starch (isolation as described above) in $6 \mathrm{mM}$ iodine solution $(0.229 \mathrm{~g}$ of re-sublimed iodine in $270 \mathrm{ml}$ of DMSO and $30 \mathrm{~mL}$ of deionised water) was left to dissolve on a vertical multifunction rotator (PTR 35, Grant BIO) providing end over end mixing to allow starch to dissolve overnight. The samples were then diluted 1:10 with deionised water and left for 30 min to allow the iodine-amylose complex to form a stable colour prior to reading the absorbance at $600 \mathrm{~nm}$ (Benchmark ${ }^{\mathrm{TM}}$ Plus Microplate Reader, Bio-Rad) against the reagent blank. The apparent amylose content was calculated using a standard curve of amylose standards (1-5 mg potato amylose type III, Sigma-Aldrich). ${ }^{33-35}$

\subsection{Non-starch polysaccharide analysis}

2.8.1. Determination of total and water-extractable monosaccharides. Briefly, for 'total' monosaccharide analysis, $5 \mathrm{mg}$ of semolina sample from WT and sbeIIa/b-AB mutant wheat lines were suspended in $10 \mathrm{~mL}$ of deionised water $(0.5 \mathrm{mg}$ $\mathrm{mL}^{-1}$ ) and homogenised in a glass/Teflon homogeniser. Triplicate aliquots of $200 \mu \mathrm{L}$ (equivalent to $100 \mu \mathrm{g}$ of the original sample) were taken and freeze-dried overnight. For WEmonosaccharide analysis $5 \mathrm{mg}$ of the semolina sample from WT and sbeIIa/b-AB wheat were resuspended in $1 \mathrm{~mL}$ of deionised water and incubated on a Denley spiromix for 30 minutes at $25{ }^{\circ} \mathrm{C}$. The suspension was centrifuged at $2500 \mathrm{~g}$ for 10 minutes and the supernatant was collected, aliquoted and freeze-dried overnight. Both TOT and WE samples were hydrolysed in $2 \mathrm{M}$ trifluoroacetic acid (TFA) as described by Bromley et al. ${ }^{36}$ Hydrolysates were diluted to $0.25 \mu \mathrm{g} \mu \mathrm{L}^{-1}$ in deionised water. If samples were not to be analysed immediately, they were stored at $-20{ }^{\circ} \mathrm{C}$. Three technical replicate samples were aliquoted and further diluted in $10 \mu \mathrm{M} 2$-deoxygalactose solution (internal standard) to a concentration of $0.05 \mu \mathrm{g} \mu \mathrm{L}^{-1}$.

Neutral monosaccharides were separated by HPAEC-PAD (using a Dionex ICS-5000+ equipped with an eluent generator with EGC $500 \mathrm{KOH}$ cartridge; Thermo Scientific, USA) with a 23.5 min run on a CarboPac PA-20 column (guard column: $3 \times$ $30 \mathrm{~mm}$, analytical column: $3 \times 150 \mathrm{~mm}$; Thermo Scientific, USA) at $30{ }^{\circ} \mathrm{C}$. Twenty-five $\mu \mathrm{L}$ of the diluted sample was injected into the column, using the following conditions: isocratic $4 \mathrm{mM} \mathrm{KOH,} 14.5 \mathrm{~min} ; 100 \mathrm{mM} \mathrm{KOH,} \mathrm{15-18} \mathrm{min;} 4 \mathrm{mM}$ $\mathrm{KOH}, 18.5-23.5 \mathrm{~min}$; the flow rate was $0.5 \mathrm{ml} \mathrm{min}^{-1}$. Authentic monosaccharide standards were subjected to the same acid hydrolysis as for the experimental samples to allow for any destruction of sugars during hydrolysis prior to the generation of calibration curves and were used for peak identification. All experimental samples were randomised prior to HPAEC analysis. Calibration standards were run at the beginning, middle and end of an analytical run cycle. All data were processed with Chromeleon analytical software (version 7.2SR4; Thermo Scientific, US) to calculate the amount of the analyte in an unknown sample using external standards/calibration curves. Total and WE-AX were calculated as the sum of xylose and arabinose minus an adjustment for arabinogalactan peptide ${ }^{37,38}$ and multiplied by 0.89 to convert monosaccharide values, for xylose and arabinose, to the polysaccharide arabinoxylan.

2.8.2. Enzyme fingerprinting. Enzymatic fingerprinting was used to measure arabinoxylan oligosaccharides (AXOS) and mixed-linked $\beta$-glucan oligosaccharides (GOS) released fol- 
lowing enzymatic digestion of semolina and freeze-dried pudding samples from the WT and sbeIIa/b-AB mutant. The method used was as described by Ordaz-Ortiz et al. (2004); ${ }^{39}$ except that recombinant enzymes were used for digestion. The method used for the identification of oligosaccharides was described by Ordaz-Ortiz \& Saulnier (2005). ${ }^{40}$ Semolina from WT and sbeIIa/b-AB mutant wheat lines were digested with endo 1,4 $\beta$-xylanase (E.C.3.2.1.8), a xylanase of the GH11 group from Neocallimastix patriciarum (PRO-E0062, Promix Limited, UK,) and endo 1,3(4) glucanase ('lichenase') (E.C.3.2.1.73) from Clostridium thermocellum NCIB 10682 (PRO-E0017, Promix Limited, UK) to digest AX and MLG, respectively. Following digestion, the samples were filtered, diluted in $10 \mu \mathrm{M}$ melibiose (internal standard), and analysed using a CarboPac PA1 analytical column (guard column: $2 \times$ $50 \mathrm{~mm} \times$ analytical column: $2 \times 250 \mathrm{~mm}$ ) on a Dionex ICS-3000 (Thermo Scientific). The peak areas of the oligosaccharides released by enzyme digestion were expressed as percentages of the total peak areas of all AXOS. Two major GOS, G3 and G4, were released by enzymatic digestion. An estimate of 'total' MLG was therefore calculated as the sum of G3 + G4 peak areas, and the G3 to G4 ratio was calculated.

\subsection{In vitro starch hydrolysis of pudding samples}

To determine the effect of the combined mutations in sbeIIa and sbeIIb genes on the intrinsic susceptibility of starch to digestion by $\alpha$-amylase, starch amylolysis was determined using an established in vitro digestion protocol. ${ }^{41}$ Puddings were prepared as described in section 2.5 (Formulation and preparation of semolina pudding). The samples were digested with porcine pancreatic $\alpha$-amylase and the digestion products were quantified using PAHBAH assay for reducing sugar determination. ${ }^{41}$ Blank values taken for each assay prior to enzyme addition $\left(Y_{0}\right)$ represent the baseline sugar value and were used to account for any endogenous sugar. Starch digestibility is expressed as $C_{90}$ representing the percentage of sbeIIa/b-AB starch digested at 90 minutes compared to the WT control. ${ }^{42}$ Logarithm of slope (LOS) analysis was used to determine starch amylolysis in the first order equation

$$
C t=C_{\infty}\left(1-e^{-k t}\right)
$$

in the logarithmic form

$$
\ln \left(\frac{\mathrm{d} C}{\mathrm{~d} t}\right)=-k t+\ln \left(C_{\infty} k\right),
$$

which gives a linear plot describing the relationship between LOS digestibility constants ( $k$ and $C_{\infty}$ ), and time of amylolysis $(t) .{ }^{43}$ Here, $C t$ represents the concentration of the digestion product at time $(t), C_{\infty}$ is the product concentration at the end of the reaction time and $k$ is the digestibility rate constant. $C_{\infty}$ can also be expressed as the percentage of starch digested over the total starch content of the sample. When endogenous sugar due to processing is present at the start of the reaction, the baseline sugar value $\left(Y_{0}\right)$ is added to $C_{\infty}$ to estimate the percentage of starch digested in time $(t) .{ }^{44}$

\subsection{In vivo glycaemic index pilot study}

2.10.1. Subjects. Twelve healthy adults were recruited from a healthy volunteer database and deemed as healthy through a screening visit with a health questionnaire. Exclusion criteria were smoking, substance abuse, significant weight change, prediabetes or diabetes, familial history of diabetes, no food allergy or intolerance and not having participated in a research trial in the last 6 months. A total of 2 participants withdrew from the study for various reasons (unable to complete the meal within $15 \mathrm{~min}$ and unable to self-finger-prick). The characteristics of the 10 participants completing the study (8 females and 2 males, age $33 \pm 2$ years, BMI $23.53 \pm 0.57 \mathrm{~kg}$ $\mathrm{m}^{-2}$, mean $\pm \mathrm{SE}$ ) can be found in Table 5 . The study flow diagram based on the Consolidated Standards of Reporting Trials (CONSORT) can be found in ESI Fig. S1.† Screening and study visits took place at the National Institute for Health Research (NIHR)/Wellcome Trust Imperial Clinical Research Facility, London. The study was approved by the West London Research Ethics Committee 2 (13/LO/0696). This study was conducted from September to November 2017 according to the Declaration of Helsinki. All participants provided written informed consent before enrolment in the study.

2.10.2. Study design. The pilot study was conducted using a two-arm double-blind randomised cross-over design, based on the standardised GI screening methodology described by Brouns et al. 2005. ${ }^{45}$ Twelve participants were randomised to four test meals: two glucose drinks (The Boots Company PLC, Nottingham, England), one WT semolina pudding and one sbeIIa/b-AB semolina pudding, given in four study visits separated by a wash-out period of $2-7$ days. ${ }^{45}$ Semolina pudding meals were accompanied by water to drink. Test foods and glucose drink were designed to provide $50 \mathrm{~g}$ of available carbohydrates. The nutritional composition of the puddings is reported in ESI Table S1. $\dagger$

Participants arrived at the research facility at 08:30 am following an overnight fast. Two fasting blood samples $(-10$ and $-5 \mathrm{~min}$ ) were collected followed by administration of the test meal (i.e. sbeIIa/b-AB or WT semolina pudding and water drink) or glucose drink. Participants were asked to consume the test meal within 15 minutes at an even pace and capillary blood samples were collected at 15, 30, 45, 60, 90, and $120 \mathrm{~min}$ after consuming a test meal or glucose drink. Blood samples were self-obtained using disposable fingerpricks (Unistix needles) and glucose readings were measured using instant glucose analysers (Hemocue, Glucose 201+, Prospect Diagnostics Ltd, Dronfield, Derbyshire). Each study visit was of the same nature.

2.10.3. Statistical analysis. Statistical analyses, graphs and incremental area under the curve (iAUC) were obtained with $\mathrm{R}^{46}$ Datasets were curated using the reshape2 package and plots were obtained using the ggplot2 package. ${ }^{4,48}$ For each participant, glycaemic index (GI) was calculated from the blood glucose response values obtained from the iAUC calculated between 0 and $120 \mathrm{~min}$ as per Brouns et al. 2005. ${ }^{49} \mathrm{GI}$ was determined as the ratio of the test food (sbeIIa/b-AB or WT) and the reference glucose drink. The primary aim of the 
study was to determine whether the GI of sbeIIa/b-AB pudding was different from the WT pudding control using a paired $t$-test. As a secondary study aim, we compared the iAUC $0-120 \mathrm{~min}$ of $s b e I I a / b$-AB and WT pudding using a paired $t$-test and the blood glucose concentrations after consuming sbeIIal $b$-AB pudding and WT pudding by linear mixed-effects analysis (lme4 package). ${ }^{50}$ The linear mixed-effects model included individual responses to the meal as the main effect, individual differences over time as random effects and changes over time due to the genotype as the fixed effect (with an interaction term). Visual inspection of the residual plots did not reveal any obvious deviations from homoscedasticity or normality and $p$-values were obtained by Satterthwaite approximation. RS, amylose and NSP concentration and composition of sbeIIal $b$-AB were compared to those of the WT control using an independent two sample $t$-test. Further details of data analysis and statistical tests are described for each experiment in the Materials \& methods sections. Data are presented as means \pm SEMs unless stated otherwise.

\section{Results}

\subsection{Semolina non-starch polysaccharide characteristics}

NSP content and composition were measured in the raw semolina from WT and sbeIIa/b-AB mutant to explore the contribution of other nutritional factors to digestibility changes and glycaemic response.

The total AX content of sbeIIa/b-AB was higher than that of the WT control in semolina (two sample $t$-test, $p=0.0069$ ), (Table 1). However, there was no difference in the water-extractable AX (WE-AX) content between sbeIIa/b-AB semolina and WT (two sample $t$-test, $p=0.32$ ).

There was no significant difference in total AXOS determined by enzymatic fingerprinting (sum of peak areas of all known AXOS peaks) in unprocessed sbeIIa/b-AB and WT semolina (two sample $t$-test, $p=0.07$ ). Enzymatic fingerprinting revealed differences in the AXOS substitution pattern between sbeIIa/b-AB semolina and WT control (Table 2). Substituted AXOS were higher in sbeIIa/b-AB semolina compared to the WT control (two sample $t$-test, $p=0.0016, p=5.679 e^{-11}$, respectively), but no significant differences in unsubstituted

Table 1 Total (TOT) and water extractable (WE) arabinoxylans (AX) of sbella/b-AB semolina compared to those of the wild-type controls (WT)

\begin{tabular}{lll}
\hline $\begin{array}{l}\text { Parameter } \\
\left(\mathrm{mg} \mathrm{g}^{-1} \text { semolina DWB }\right)\end{array}$ & $\begin{array}{l}\text { Semolina WT } \\
\text { control }\end{array}$ & $\begin{array}{l}\text { Semolina } \\
\text { sbelIa } / b-\mathrm{AB}\end{array}$ \\
\hline WE-AX & $1.17 \pm 0.11$ & $1.51 \pm 0.51$ \\
TOT-AX & $13.27 \pm 0.76$ & $20.72 \pm 1.76$
\end{tabular}

Means \pm sd of independently analysed semolina samples $(n=3)$ from bulked semolina. Total and WE-AX were calculated as the sum of xylose and arabinose minus an adjustment for arabinogalactan peptide as described in ref. 38, 51 and 52, and multiplied by 0.89 to convert monosaccharide values, for xylose and arabinose, to the polysaccharide arabinoxylan. Complete monosaccharide profiles are reported in ESI Table S3.†
Table 2 AXOS of sbella/b-AB semolina compared to that of the wildtype control

\begin{tabular}{lcc}
\hline $\begin{array}{l}\text { Parameter } \\
\text { (sum of peaks relative } \\
\text { to the internal standard) }\end{array}$ & $\begin{array}{l}\text { Semolina } \\
\text { WT control }\end{array}$ & $\begin{array}{l}\text { Semolina } \\
\text { sbeIIa/b-AB }\end{array}$ \\
\hline TOT-AXOS & $10.02 \pm 0.74$ & $12.64 \pm 0.97$ \\
AXOS unsubstituted & $5.39 \pm 0.6$ & $6.61 \pm 0.79$ \\
AXOS substituted & $4.63 \pm 0.15$ & $6.03 \pm 0.2$ \\
MLG (G3 + G4) & $2.29 \pm 0.12$ & $2.78 \pm 0.11$
\end{tabular}

Means \pm SEMs of independently analysed semolina samples $(n=4)$ from bulked semolina.

AXOS were detected (two sample $t$-test, $p=0.26$ ). To describe the structure of MLG and the distribution of $\beta 1-3$ and $\beta 1-4$ bonds, an estimate of total MLG was obtained by summing G3 and G4 GOS peak areas and the ratio of G3 to G4 GOS determined as the ratio of G3 to G4 GOS peak area. ${ }^{18}$ The mean of $\mathrm{G} 3+\mathrm{G} 4$ of sbeIIa/b-AB semolina was higher than that of the WT control (two sample $t$-test, $p=0.028$ ) and the ratio of G3 to G4 was 2.4 for sbeIIa/b-AB semolina vs. 2.2 for the WT control. NSP content and composition were also measured for freezedried pudding prepared alongside pudding portions served in the GI pilot study described below; these results can be found in the ESI (Tables S4 and S5†).

\subsection{Starch characteristics before and after processing}

The TS contents of bulked sbeIIa/b-AB and WT control semolina were $60.65 \pm 3.84 \mathrm{~g}$ per $100 \mathrm{~g}$ on a dry weight basis (DWB) and $67.90 \pm 1.44 \mathrm{~g}$ per $100 \mathrm{~g}$, respectively (unpaired twosample $t$-test, $p=0.11$ ). The amylose content of starch isolated from sbeIIa/b-AB semolina was $33.05 \% \pm 0.69 \%(\mathrm{w} / \mathrm{w}$ dry matter basis) while the WT control was $23.11 \% \pm 1.5 \%(\mathrm{w} / \mathrm{w}$ dry weight basis) (unpaired two-sample $t$-test, $p=0.004$ ).

Volume moment mean $\mathrm{D}[4,3]$ particle size of sbeIIa/b-AB semolina was larger compared to that of the WT control (means \pm SEMs were $279 \pm 4.23 \mu \mathrm{m}$ and $258.42 \pm 5.54 \mu \mathrm{m}$, respectively, $n=3$ of bulked semolina, unpaired two sample $t$-test, $p=0.004)$. The particle size distribution is shown in ESI Fig. S2. $\dagger$

The resistant starch content of raw sbeIIa/b-AB semolina $(4.87 \pm 0.6 \mathrm{~g}$ per $100 \mathrm{~g}$ starch $\mathrm{DWB})$ was higher than that of the WT control $(0.77 \pm 0.34 \mathrm{~g}$ per $100 \mathrm{~g}$ starch DWB, two sample $t$-test, $p=0.004)$. Different amounts of resistant starch were detected after cooking ('gelatinisation') and cooling ('retrogradation') of sbeIIa/b-AB and WT semolina, as shown in Fig. 1. sbeIIa/b-AB semolina was characterised by a higher proportion of resistant starch than the WT control when gelatinised and retrograded.

\subsection{In vitro starch digestibility of pudding}

Mutant sbeIIa/b-AB pudding was less digested overall, and digestion occurred at a lower rate compared to the WT control. Amylolysis profiles of sbeIIa/b-AB and WT control are shown in Fig. 2a. sbeIIa/b-AB pudding starch was significantly less digested after 90 minutes, compared to the WT control 

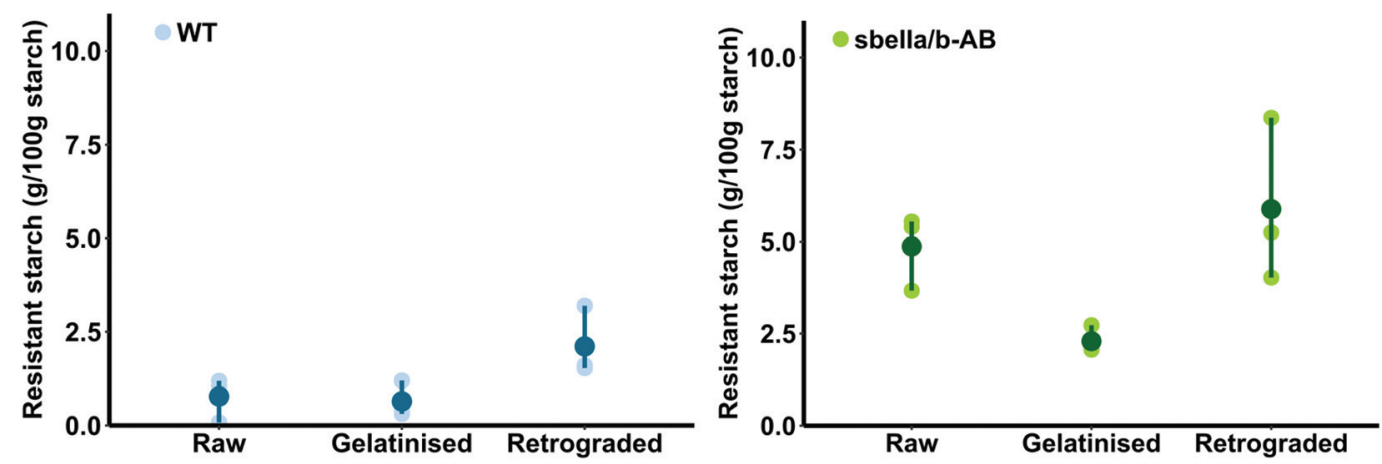

Fig. 1 Proportion of resistant starch (expressed as the amount of RS per $100 \mathrm{~g}$ of semolina starch) of raw, cooked (gelatinised) and cooled (retrograded) semolina. Means \pm SEMs comparing sbella/b-AB to WT within each treatment. Dark blue and dark green datapoints in the figure represent means of sbella/b-AB and WT for each treatment. Light blue and light green datapoints represent $n=3$ replicates of bulked semolina that were extracted and treated independently.
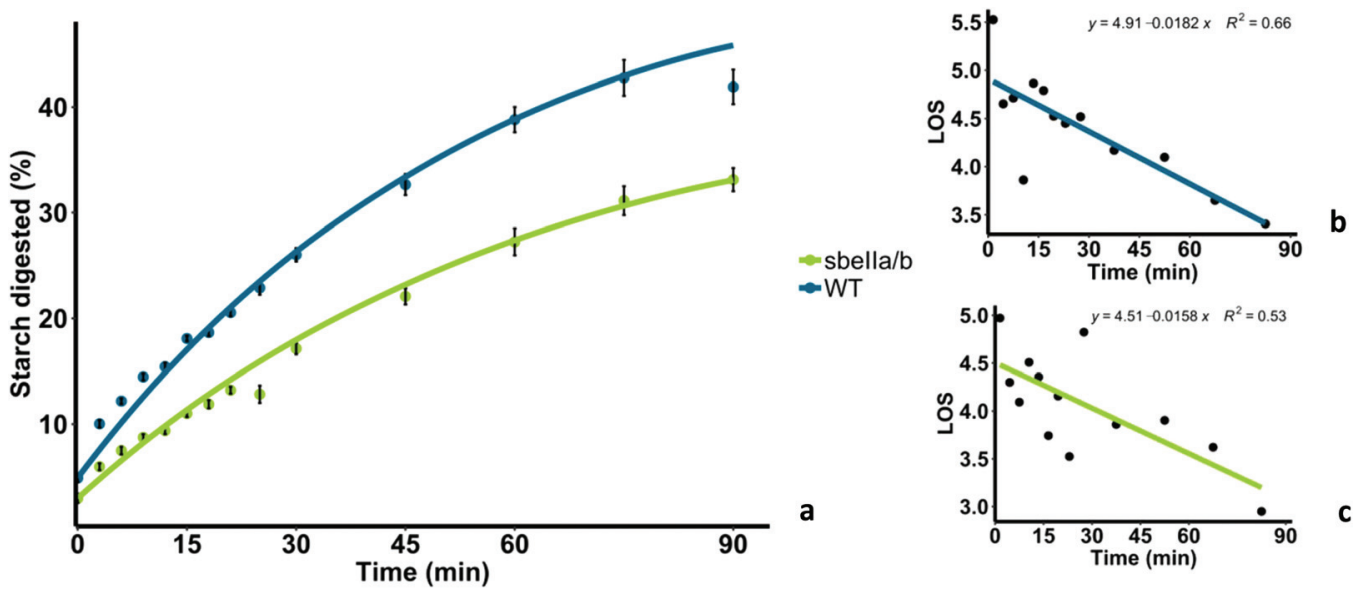

Fig. 2 (a) Starch digestibility of sbella/b-AB pudding (sbella/b) and wild-type pudding (WT), and starch amylolysis experimental data are shown by fitting a first-order equation based on the $k$ and $C_{\infty}$ values obtained from the LOS analysis. Each experimental data point represents the mean value from the analysis of three pudding samples with error bars $= \pm$ SEMs. (b) LOS plot obtained for WT pudding. (c) LOS plot for sbella/b-AB pudding. The linear phase is defined using eqn (2) from which $k$ and $C_{\infty}$ values can be estimated.

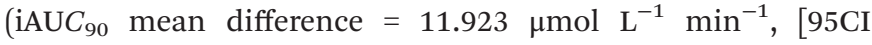
$44646.60,71756.06]$, paired $t$-test, $p=0.0002839$ ). LOS analysis was used to determine the digestion rate at 90 minutes and both the starch amylolysis rate and extent were found to be lower for sbeIIa/b-AB pudding compared to those for the WT control $\left(C_{\infty}\right.$ and $\left.k\right)$. Linear plots with single rate constants were obtained from LOS analysis (Fig. 2b and c).

Table 3 Variable estimates from the digestibility curves and LOS analysis of sbella/b-AB pudding compared to those of WT control pudding

\begin{tabular}{llll}
\hline Pudding & $C_{90}(\%)$ & $C_{\infty}(\%)$ & $k\left(\mathrm{~min}^{-1}\right)$ \\
\hline WT control & 47.38 & 50.9 & 0.0209 \\
sbeIIa/b-AB & 33.29 & 39.76 & 0.0158
\end{tabular}

$C_{90}$ is the proportion of starch digested by $\alpha$-amylase after $90 \mathrm{~min}$; this is obtained from the starch digestibility curve. $C_{\infty}$ is the total extent of starch amylolysis for each digestive phase accounting for $Y_{0}$ (endogenous baseline sugar) and $k$ is the rate constant of the reaction; both parameters are obtained applying the LOS analysis to the digestibility data.
The total breakdown of starch represented by $C_{\infty}$ and the rate constant $(k)$ of starch digestion were determined from the slope and $y$-intercept of the LOS plot and are reported in Table 3. Both $C_{\infty}$ and $k$ were lower for sbeIIa/b-AB pudding than the WT control. These kinetic parameters provided a good fit to experimental data as shown in Fig. 2a: however, the low $R^{2}$ values lower our confidence in the accuracy of the rate of digestion measured by the parameter $k$.

\subsection{In vivo glycaemic index of pudding}

Test meal characteristics are reported on a dry weight basis (DWB) in Table 4. The average pudding portion was $415.3 \mathrm{~g} \pm$ $12.9 \mathrm{~g}$ (mean $\pm \mathrm{SEM}$ of 10 puddings served) for sbeIIa/b-AB with $85.1 \%$ moisture content and $384.95 \mathrm{~g} \pm 7.5 \mathrm{~g}$ (mean \pm SEM of 10 puddings served) for WT with $83.6 \%$ moisture content.

Mutant sbeIIa/b-AB pudding had more resistant starch compared to WT pudding (Welch two sample $t$-test, $p=0.0005$ ). The total starch content of WT pudding was found to be $2.3 \mathrm{~g}$ 
Table 4 Test meals characteristics

\begin{tabular}{llll}
\hline & $\begin{array}{l}\text { Semolina (g per } \\
\text { Pudding }\end{array}$ & $\begin{array}{l}\text { TS (g per } \\
\text { serving, DWB) }\end{array}$ & $\begin{array}{l}\text { RS (g per } \\
\text { serving, DWB) }\end{array}$ \\
\hline WT control & 63.14 & 43.272 & 2.505 \\
sbeIIa/b-AB & 61.76 & 45.355 & 4.503
\end{tabular}

Semolina per pudding serving and starch characteristics of the pudding served on a DWB. TS and RS are calculated from the semolina starch content and adjusted for the measured moisture content of the pudding.

Table 5 Characteristics of the 10 participants completing the study

\begin{tabular}{ll}
\hline Males/females $(n)$ & $2 / 8$ \\
\hline Age $(\mathrm{y})$ & $33 \pm 2$ \\
Height $(\mathrm{cm})$ & $154 \pm 17$ \\
Weight $(\mathrm{kg})$ & $69 \pm 3$ \\
BMI $\left(\mathrm{kg} \mathrm{m}^{-2}\right)$ & $23.5 \pm 0.6$ \\
Ethnicity & White Caucasian (7) \\
& Southwest Asian (3) \\
Fasting glucose $\left(\mathrm{mmol} \mathrm{L}^{-1}\right)$ & $4.7 \pm 0.07$ \\
Reference AUC $\left(\mathrm{mmol} \mathrm{min}^{-1}\right)$ & $198 \pm 25$ \\
Time taken to eat the test meal (min) & $11.8 \pm 1.4$ \\
& \\
Means \pm SEMs. &
\end{tabular}

lower than that of the sbeIIa/b-AB pudding, possibly due to unexpected water losses during cooking (Welch two sample $t$-test, $p=0.0347)$. The test meals were consumed within approximately 12 minutes. A total of ten participants completed the study and their characteristics are shown in Table 5.

The mean GI and iAUCs of sbeIIa/b-AB and WT control puddings are reported in Table 6, and the capillary blood glucose concentrations of 10 participants who completed the study are reported in ESI Table S2. $\dagger$ We found no evidence of difference in GI (mean difference $=-13.9$ [95CI $-17.00758,44.80758]$, paired $t$-test, $p=0.335$ ). The blood glucose profile after consuming sbeIIa/b-AB pudding (iAUC 0-120 min) did not significantly differ from the WT control (mean difference $=-24.019$ [95CI $-23.46,71.49]$, paired $t$-test, $p=0.282$ ), Fig. $3 \mathrm{~b}$. No main effect of sbeIIa/b-AB pudding was found for reducing blood glucose concentration (linear mixed-effects model, $p=$ 0.37011); however 60 minutes after consumption, sbeIIa/b-AB pudding resulted in a lower blood glucose concentration. This may suggest that the effect of the pudding genotype on blood glucose concentration is dependent on time, an interaction (time $\times$ genotype) which is significant at 60 minutes (linear

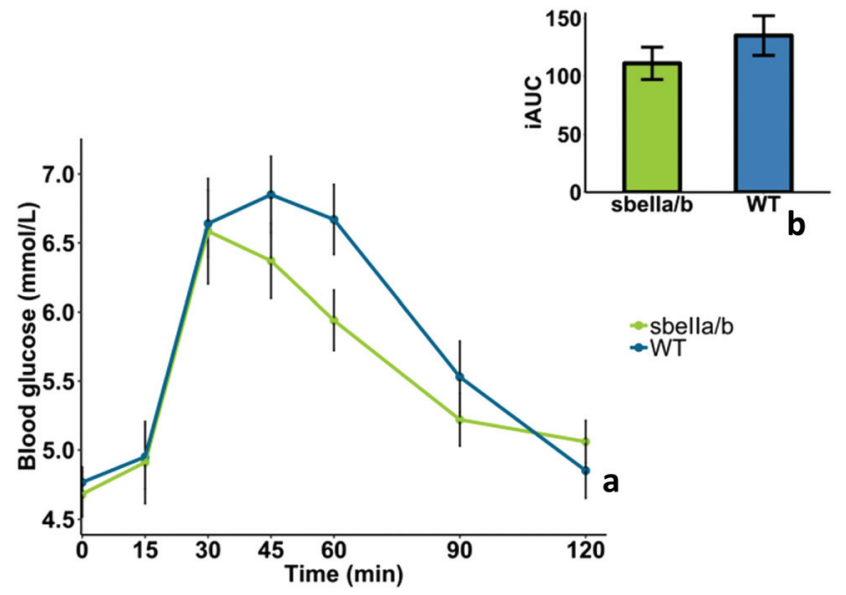

Fig. 3 (a) Postprandial changes in blood glucose concentration after sbella/b-AB (sbella/b) and wild-type (WT) control pudding meals. (b) iAUC blood glucose. Values are mean deviations from baseline \pm SEMs ( $n=10$ individuals).

mixed-effects model, $p=0.042$ ) but not overall (linear mixedeffects model, $p=0.07$ ), Fig. 3 a.

\section{Discussion}

Our study features a new approach that combines modified starch structures in raw materials with optimised food processing to design healthier starchy foods with steady glucose release. The sbeIIa/b-AB mutant wheat semolina used in this study was previously characterised by high-amylose and resistant starch concentrations. However, it was not known to what extent the desirable nutritional properties of sbeIIa/b-AB mutant wheat were preserved after processing the wheat into a food (in this case, semolina pudding), nor if these properties would impact in vivo glycaemic responses. In this study, we explored starch resistance to digestion in vitro after hydrothermal processing of semolina into a pudding and its GI in a pilot study in vivo. We also reported the NSP content and composition of sbeIIa/b-AB wheat in raw semolina for the first time.

In vitro digestion kinetic analysis of sbeIIa/b-AB pudding showed lower starch digestibility (iAU $C_{90}$ ), starch breakdown $\left(C_{\infty}\right)$ and lower rate of digestion $(k)$ at 90 minutes compared to the WT control. A lower in vitro starch digestibility would be expected to coincide with a lower glucose response in vivo. ${ }^{42}$ Previous studies have shown a direct correlation between the

Table 6 Blood glucose responses after test meals

\begin{tabular}{lllll}
\hline Meals & $\begin{array}{l}\text { Glucose iAUC mmol L } \\
(0-30 \mathrm{~min})\end{array}$ & $\begin{array}{l}\text { Glucose iAUC mmol L } \\
(0-60 \mathrm{~min})\end{array}$ & $\begin{array}{l}\text { Glucose iAUC } \mathrm{mmol} \mathrm{L}^{-1} \mathrm{~min}^{-1} \\
(0-120 \mathrm{~min})\end{array}$ & $\begin{array}{l}\mathrm{Glycaemic}^{-1} \\
\text { index }\end{array}$ \\
\hline WT control & $18.4 \pm 2.6$ & $78.1 \pm 7.2$ & $135.0 \pm 17.1$ & $73.85 \pm 12$ \\
sbeIIa/b-AB & $19.2 \pm 4.2$ & $68.3 \pm 10$ & $111.0 \pm 14$ & $59.78 \pm 7.6$
\end{tabular}

Values are means \pm SEM, $n=10$ individuals. 
digestibility index $C_{90}$ obtained from starch digestibility curves and GI values measured in vivo. The GI difference between sbeIIa/b-AB and WT puddings was not statistically significant but it was consistent with the starch digestibility measured in vitro $\left(C_{90}\right)$. Unexpected water losses during cooking may have affected the accuracy of the glycaemic load delivered, as the TS content of the pudding servings did not match as anticipated. Although the overall GI values were not statistically different, there was a tendency for lower blood glucose values at 60 minutes after consumption of sbeIIa/b-AB pudding. This suggests that while the cooking method may need to be optimised to exploit the potential of the higher RS content in lowering glucose response, consumption of sbeIIal $b$-AB wheat-based food products may contribute to lower postprandial glycaemia in healthy subjects. Future studies on larger cohorts should focus on measuring the extent of the reduction in postprandial glycaemia.

It is worth noting that the RS content of unprocessed sbeIIa/b-AB semolina was lower than that reported in previous studies using the same genotypes. ${ }^{24}$ The lower RS concentration in this batch of sbeIIa/b-AB semolina may have resulted from the environmental effects during the growth of the wheat. ${ }^{57}$ However, it does suggest that the RS concentration in the pudding alone administered in this pilot study may not be sufficient to elicit a lower glycaemic response compared to the WT control. While the use of wheat with a higher content of RS may have been preferable, the authors worked with the available material which was considered to be satisfactory to develop novel durum wheat foods and to explore its effect on glycaemic response.

We observed that RS remained higher in sbeIIa/b-AB compared to the WT after cooking semolina into a pudding which was likely due to a combination of factors including starch retrogradation and starch native structure (amylose). sbeIIa/b-AB wheat starch may be more prone to gelatinisation than WT wheat starch. Li and colleagues characterised starch physicochemical properties of a range of wheat lines with different amylose concentrations by combining multiple alleles of $S B E I I$ genes. They reported lower gelatinization enthalpy $(\Delta H)$ and greater gelatinization range for high amylose mutant starches compared to the corresponding wild-type control. However, the bread wheat lines analysed by Li et al. had considerably a higher amylose content, ranging from 36\% (WT) to $93 \%$ in line RS100. ${ }^{53}$ Because of the relatively small difference in amylose concentration between sbeIIa/b-AB and WT wheat starch described in this study, a dramatic difference in the degree of gelatinisation seems unlikely.

Cooked and cooled ('retrograded') sbeIIa/b-AB semolina had $\sim 2.5$-fold more RS compared to the WT control. In this study, starch resistance to digestion was mainly determined by the structural and physical characteristics of raw semolina which influenced the properties of the surrounding food matrix. ${ }^{54,55}$ Milling of sbeIIa/b-AB and WT wheat grains resulted in sbeIIa/ $b$-AB semolina with an $\sim 8 \%$ larger particle size compared to WT semolina. Durum wheat is generally milled to semolina grade, a coarse particle size, to reduce the high starch damage due to the kernel hardness. ${ }^{56}$ Particle size has previously been shown to have influenced starch gelatinisation, bioaccessibility and postprandial glycaemic responses to starch-based foods. ${ }^{57}$ However, the differences in the size of the fractions used in previous studies were three orders of magnitude larger than the subtle $21 \mu \mathrm{m}$ difference in the size between mutant sbeIIal $b$-AB and WT particles used in this study. In this case, particle size difference is unlikely to be a contributing mechanism to the difference in digestibility observed..$^{15}$ The milling process was carried out under the same conditions for both WT and sbeIIa/b-AB. Because of the novelty of sbeIIa/b-AB wheat, we did not anticipate that milling sbeIIa/b-AB wheat grains would result in sbeIIa/b-AB semolina with a larger particle size. This is possibly one of the characteristics of sbeIIa/b-AB semolina which will need to be taken into account when using sbeIIal $b$-AB durum wheat for food formulations.

sbeIIa/b-AB semolina used in this study was characterised by increased AX and MLG contents, derived from cell wall components. It has been suggested that under certain conditions (such as high molecular weight and high concentration) some NSP components may influence the viscosity of a food matrix and therefore alter the interaction between digestive enzymes and substrates. ${ }^{21,22}$ A higher ratio of G3 and G4 residues in sbeIIa/b-AB semolina compared to that in the WT control suggests a change in the MLG structure in sbeIIa/b-AB wheat. However, the increase in the MLG content (G3 + G4) and the change in the structure (G3:G4) measured in raw sbeIIa/b-AB semolina are still within the range indicated by De Santis et al. for other durum wheat cultivars. ${ }^{58}$ The total AX content of sbeIIa/b-AB semolina was higher than that of the WT control and we also observed an increased proportion of substituted AXOS. As AX solubility depends largely on their molecular weight and substitution degree, ${ }^{59}$ a change in AX composition could correspond to a change in viscosity properties. However, due to the relatively small increase in the NSP content, it is unlikely that pudding viscosity was affected by these changes. Increases in NSP concentrations are of interest as they are known to exert health benefits via mechanisms linked to gut fermentation (prebiotic activity). ${ }^{60}$ Overall, the difference in NSP concentrations in sbeIIa/b-AB wheat is an interesting observation and future studies should take into account their potential role in gut fermentation.

This study essentially provides new hypotheses regarding the applicability of high resistant starch semolina for food production and its potential effects on glucose metabolism. It is also important to consider that pilot studies are usually used to determine the feasibility of a study rather than test a hypothesis. For this reason we did not base our pilot study on a calculated sample size, instead we relied on a well standardised method for GI screening as described by Brouns et al. $2005^{45}$ which suggests that the inclusion of 10 subjects provides a reasonable degree of power and precision to measure the GI. Furthermore, the use of the sbeIIa/b-AB semolina described in the study has helped the authors to fine-tune the RS concentration and formulation of wheat foods, which will 
be tested in larger follow-up studies to measure the impact of sbeIIa/b-AB wheat on glucose response.

\section{Conclusions}

This is the first time that the glycaemic response to $s b e I I a / b-\mathrm{AB}$ wheat has been studied within a processed food matrix. In this pilot study, semolina puddings were prepared from WT control and sbeIIa/b-AB mutant wheat harvested in bulk and nutritionally relevant properties were examined both in vitro and in vivo. Using reverse genetic approaches to fine-tune the digestibility of complex carbohydrates in staple crops is becoming a widely accepted method to optimise energy release and improve the nutritional profile of staple foods. We have shown that RS and nutritional properties are influenced by processing. Despite the high RS concentration of sbeIIa/b-AB wheat, the GI of sbeIIa/b-AB pudding was not significantly different from that of WT control pudding. Nevertheless, there are likely advantages of sbeIIa/b-AB wheat such as lower glycaemic load, and increased RS and fibre intake. Future studies should focus on optimising the processing conditions of wheat-based foods with high RS and NSP concentrations. The present study highlights the importance of studying novel wheat with improved nutritional traits in the context of a food matrix to determine its potential impact on health. Further larger studies in vivo are needed to determine the physiological effect of sbeIIa/b-AB wheat foods and their potential to boost the dietary intake of RS.

\section{Abbreviations}

$\begin{array}{ll}\text { RS } & \text { Resistant starch } \\ \text { WT } & \text { Wild-type } \\ \text { DWB } & \text { Dry weight basis } \\ \text { GI } & \text { Glycaemic index } \\ \text { iAUC } & \text { Incremental area under the curve } \\ \text { NSPs } & \text { Non-starch polysaccharides } \\ \text { AX } & \text { Arabinoxylans } \\ \text { TOT-AX } & \text { Total arabinoxylans } \\ \text { WE-AX } & \text { Water extractable arabinoxylans } \\ \text { TOT-AXOS } & \text { Sum of arabinoxylan oligosaccharides } \\ \text { MLG } & \text { Mixed-linkage } \beta \text {-glucans }\end{array}$

\section{Conflicts of interest}

None of the authors reported a conflict of interest related to the study.

\section{Acknowledgements}

We thank Dr George Savva (QIB) for statistical guidance, Prof. Peter Wilde (QIB), Dr Cristobal Uauy (JIC), Dr Frederick
Warren (QIB) and Dr Peter Ryden (QIB) for helpful discussions.

Infrastructure support was provided by the NIHR Imperial Biomedical Research Centre and the NIHR Imperial Clinical Research Facility, London (UK). The views expressed are those of the authors and not necessarily those of the NHS, the NIHR or the Department of Health and Social Care. The authors would like to thank all volunteers involved in the study.

The authors' responsibilities were as follows: MC, ACM, EC, AP and AW carried out the experiments. MC and EC processed the experimental data and performed the analysis. MC wrote the manuscript with support from CHE, BAH, AL and GSF. $\mathrm{CHE}$ and $\mathrm{BAH}$ supervised the project. All authors discussed the results and commented on the manuscript.

This work was supported by the UKRI Biotechnology and Biological Sciences Research Council Norwich Research Park Biosciences Doctoral Training Partnership grant number BB/ M011216/1. The authors gratefully acknowledge the support of the BBSRC Institute Strategic Programme Food Innovation and Health BB/R012512/1 and its constituent projects $\mathrm{BBS} / \mathrm{E} / \mathrm{F} /$ 000PR10343 (Theme 1, Food Innovation) and $\mathrm{BBS} / \mathrm{E} / \mathrm{F} /$ 000PR10345 (Theme 2, Digestion in the Upper GI Tract) and previously by the Institute Strategic Programme Grant ('Food and Health'; Grant No. BB/J004545/1) to the Institute of Food Research. The authors would also like to acknowledge the support of the BBSRC Institute Strategic Programme Grants 'Molecules from Nature' - Crop Quality BBS/E/J/000PR9799 to the John Innes Centre and 'Designing Future Wheat' (BB/ P016855/1) to Rothamsted Research and the John Innes Centre.

\section{References}

1 PHE, National Diet and Nutrition Survey 2014/2015 to 2015/2016, https:/www.gov.uk/government/statistics/ndnsresults-from-years-7-and-8-combined.

2 F. B. $\mathrm{Hu}$, Are refined carbohydrates worse than saturated fat ? 1-3, Am. J. Clin. Nutr., 2010, 91, 1541-1542.

3 F. B. Hu and W. C. Willett, Optimal Diets for Prevention of Coronary Heart Disease, J. Am. Med. Assoc., 2002, 288, 2569-2569.

4 G. Frost and A. Dornhorst, The relevance of the glycaemic index to our understanding of dietary carbohydrates, Diabetic Med., 2000, 17, 336-345.

5 J. H. Cummings, M. B. Roberfroid, H. Andersson, C. Barth, A. Ferro-Luzzi, Y. Ghoos, M. Gibney, K. Hermonsen, W. James, O. Korver, D. Lairon, G. Pascal and A. Voragen, A new look at dietary carbohydrate: chemistry, physiology and health JH Cummings, MB Roberfroid and members of the Paris Carbohydrate Group, Eur. J. Clin. Nutr., 1997, 51, 417-423.

6 C. S. Byrne, E. S. Chambers, D. J. Morrison and G. Frost, The role of short chain fatty acids in appetite regulation and energy homeostasis, Int. J. Obes., 2015, 39, 1331-1338. 
7 A. Buléon, P. Colonna, V. Planchot and S. Ball, Starch granules: structure and biosynthesis, Int. J. Biol. Macromol., 1998, 23, 85-112.

8 F. J. Warren, B. Zhang, G. Waltzer, M. J. Gidley and S. Dhital, The interplay of $\alpha$-amylase and amyloglucosidase activities on the digestion of starch in in vitro enzymic systems, Carbohydr. Polym., 2015, 117, 192-200.

9 H. Goesaert, K. Brijs, W. S. Veraverbeke, C. M. Courtin, K. Gebruers and J. A. Delcour, Wheat flour constituents: how they impact bread quality, and how to impact their functionality, Trends Food Sci. Technol., 2005, 16, 12-30.

10 R. C. Eerlingen, H. Jacobs and J. A. Delcour, Enzyme-Resistant Starch. V. Effect of Retrogradation of Waxy Maize Starch on Enzyme Susceptibility, Cereal Chem., 1994, 71, 351-355.

11 R. Cui and C. G. Oates, The effect of retrogradation on enzyme susceptibility of sago starch, Carbohydr. Polym., 1997, 32, 65-72.

12 H. Li, M. J. Gidley and S. Dhital, High-Amylose Starches to Bridge the "Fiber Gap": Development, Structure, and Nutritional Functionality, Compr. Rev. Food Sci. Food Saf., 2019, 18, 362-379.

13 A. Htoon, A. K. Shrestha, B. M. Flanagan, A. Lopez-Rubio, A. R. Bird, E. P. Gilbert and M. J. Gidley, Effects of processing high amylose maize starches under controlled conditions on structural organisation and amylase digestibility, Carbohydr. Polym., 2009, 75, 236-245.

14 C. Hoebler, A. Karinthi, H. Chiron, M. Champ and J. L. Barry, Bioavailability of starch in bread rich in amylose: metabolic responses in healthy subjects and starch structure, Eur. J. Clin. Nutr., 1999, 53, 360-366.

15 C. H. Edwards, M. M. Grundy, T. Grassby, D. Vasilopoulou, G. S. Frost, P. J. Butterworth, S. E. Berry, J. Sanderson and P. R. Ellis, Manipulation of starch bioaccessibility in wheat endosperm to regulate starch digestion, postprandial glycemia, insulinemia, and gut hormone responses: a randomized controlled trial in healthy ileostomy participants 1,2, Am. J. Clin. Nutr., 2015, 102, 791-800.

16 J. M. Lattimer and M. D. Haub, Effects of Dietary Fiber and Its Components on Metabolic Health, Nutrients, 2010, 2, 1266-1289.

17 H. N. Englyst and S. M. Kingman, Dietary fiber and resistant starch - A nutritional characterisation of plant polysaccharides, Diet. Fiber, 1987, 49-65.

18 M. A. De Santis, O. Kosik, D. Passmore, Z. Flagella, P. R. Shewry and A. Lovegrove, Comparison of the dietary fibre composition of old and modern durum wheat (Triticum turgidum spp. durum) genotypes, Food Chem., 2018, 244, 304-310.

19 P. R. Shewry, M. J. Hawkesford, V. Piironen, A.-M. Lampi, K. Gebruers, D. Boros, A. A. M. Andersson, P. Åman, M. Rakszegi, Z. Bedo and J. L. Ward, Natural Variation in Grain Composition of Wheat and Related Cereals, J. Agric. Food Chem., 2013, 61, 8295.

20 D. J. Mares and B. A. Stone, Studies on wheat endosperm i. Chemical composition and ultrastructure of the cell walls, Aust. J. Biol. Sci., 1973, 26, 793-812.
21 M. M.-L. Grundy, C. H. Edwards, A. R. Mackie, M. J. Gidley, P. J. Butterworth and P. R. Ellis, Re-evaluation of the mechanisms of dietary fibre and implications for macronutrient bioaccessibility, digestion and postprandial metabolism, Br. J. Nutr., 2016, 116, 816-833.

22 L. Saulnier, N. Peneau and J. F. Thibault, Variability in grain extract viscosity and water-soluble arabinoxylan content in wheat, J. Cereal Sci., 1995, 22, 259-264.

23 I. J. Tetlow and M. J. Emes, A review of starch-branching enzymes and their role in amylopectin biosynthesis, IUBMB Life, 2014, 66, 546-558.

24 B. Hazard, X. Zhang, P. Colasuonno, C. Uauy, D. M. Beckles and J. Dubcovsky, Induced mutations in the starch branching enzyme II (SBEII) genes increase amylose and resistant starch content in durum wheat, Crop Sci., 2012, 52, 1754-1766.

25 B. Hazard, X. Zhang, M. Naemeh, M. K. Hamilton, B. Rust, H. E. Raybould, J. W. Newman, R. Martin and J. Dubcovsky, Mutations in Durum Wheat SBEII Genes affect Grain Yield Components, Quality, and Fermentation Responses in Rats, Crop Sci., 2015, 6, 2813-2825.

26 A. R. Bird and A. Regina, High amylose wheat: A platform for delivering human health benefits, J. Cereal Sci., 2018, 82, 99-105.

27 C. Souza da Silva, D. Haenen, S. J. Koopmans, G. J. E. J. Hooiveld, G. Bosch, J. E. Bolhuis, B. Kemp, M. Müller and W. J. J. Gerrits, Effects of resistant starch on behaviour, satiety-related hormones and metabolites in growing pigs, Animal, 2014, 8, 1402-1411.

28 P. R. Regmi, T. A. T. G. van Kempen, J. J. Matte and R. T. Zijlstra, Starch with High Amylose and Low in Vitro Digestibility Increases Short-Chain Fatty Acid Absorption, Reduces Peak Insulin Secretion, and Modulates Incretin Secretion in Pigs, J. Nutr., 2011, 141, 398-405.

29 H. J. Willis, A. L. Eldridge, J. Beiseigel, W. Thomas and J. L. Slavin, Greater satiety response with resistant starch and corn bran in human subjects, Nutr. Res., 2009, 29, 100-105.

30 J. Harrold, L. Breslin, J. Walsh, J. Halford and C. Pelkman, Satiety effects of a whole-grain fibre composite ingredient: Reduced food intake and appetite ratings, Food Funct., 2014, 5, 2574-2581.

31 B. Hazard, X. Zhang, M. Naemeh and J. Dubcovsky, Registration of Durum Wheat Germplasm Lines with Combined Mutations in SBEIIa and SBEIIb Genes Conferring Increased Amylose and Resistant Starch, J. Plant Regist., 2014, 8, 334-338.

32 T. Verhoeven, B. Fahy, M. Leggett, G. Moates and K. Denyer, Isolation and characterisation of novel starch mutants of oats, J. Cereal Sci., 2004, 40, 69-79.

33 C. A. Knutson, Evaluation of variations in amylose-iodine absorbance spectra, Carbohydr. Polym., 2000, 42, 65-72.

34 C. A. Knutson and M. J. Grove, Rapid Method for Estimation of Amylose in Maize Starches, Cereal Chem., 0094, 71, 469-471.

35 C. A. Knutson, A Simplified Colorimetric Procedure for Determination of Amylose in Maize Starches, Cereal Chem., 1986, 63, 89-92. 
36 J. R. Bromley, M. Busse-Wicher, T. Tryfona, J. C. Mortimer, Z. Zhang, D. M. Brown and P. Dupree, GUX1 and GUX2 glucuronyltransferases decorate distinct domains of glucuronoxylan with different substitution patterns, Plant J., 2013, 74, 423-434.

37 N. Anders, M. D. Wilkinson, A. Lovegrove, J. Freeman, T. Tryfona, T. K. Pellny, T. Weimar, J. C. Mortimer, K. Stott, J. M. Baker, M. Defoin-Platel, P. R. Shewry, P. Dupree and R. A. C. Mitchell, Glycosyl transferases in family 61 mediate arabinofuranosyl transfer onto xylan in grasses, Proc. Natl. Acad. Sci. U. S. A., 2012, 109, 989-993.

38 N. Anders, M. D. Wilkinson, A. Lovegrove, J. Freeman, T. Tryfona, T. K. Pellny, T. Weimar, J. C. Mortimer, K. Stott, J. M. Baker, M. Defoin-Platel, P. R. Shewry, P. Dupree and R. A. C. Mitchell, Glycosyl transferases in family 61 mediate arabinofuranosyl transfer onto xylan in grasses Supporting Information, Proc. Natl. Acad. Sci. U. S. A., 2012, 1-10, DOI: 10.1021/jo101696z.

39 J. J. Ordaz-Ortiz, F. Guillon, O. Tranquet, G. Dervilly-Pinel, V. Tran and L. Saulnier, Specificity of monoclonal antibodies generated against arabinoxylans of cereal grains, Carbohydr. Polym., 2004, 57, 425-433.

40 J. J. Ordaz-Ortiz, M. F. Devaux and L. Saulnier, Classification of wheat varieties based on structural features of arabinoxylans as revealed by endoxylanase treatment of flour and grain, J. Agric. Food Chem., 2005, 53, 8349-8356.

41 C. H. Edwards, M. Maillot, R. Parker and F. J. Warren, A comparison of the kinetics of in vitro starch digestion in smooth and wrinkled peas by porcine pancreatic alphaamylase, Food Chem., 2018, 244, 386-393.

42 C. H. Edwards, N. Cochetel, L. Setterfield, N. Perez-Moral and F. J. Warren, A single-enzyme system for starch digestibility screening and its relevance to understanding and predicting the glycaemic index of food products, Food Funct., 2019, 10, 4751-4760.

43 C. H. Edwards, F. J. Warren, P. J. Milligan, P. J. Butterworth and P. R. Ellis, A novel method for classifying starch digestion by modelling the amylolysis of plant foods using first order enzyme kinetic principles, Food Funct., 2014, 5, 27512758.

44 P. J. Butterworth, F. J. Warren, T. Grassby, H. Patel and P. R. Ellis, Analysis of starch amylolysis using plots for first-order kinetics, Carbohydr. Polym., 2012, 87, 2189-2197.

45 F. Brouns, I. Bjorck, K. N. Frayn, A. L. Gibbs, V. Lang, G. Slama and T. M. S. Wolever, Glycaemic index methodology, Nutr. Res. Rev., 2005, 18, 145-171.

46 R Core Team, R: A language and environment for statistical computing, R Foundation for Statistical Computing, Vienna, Austria, 2014, http://www.R-project.org/.

47 H. Wickham, ggplot2: Elegant Graphics for Data Analysis, Springer-Verlag, New York, 2016.

48 H. Wickham, Reshaping Data with the reshape Package, J. Stat. Software, 2007, 21, 1-20.
49 F. Brouns, I. Bjorck, K. N. Frayn, A. L. Gibbs, V. Lang, G. Slama and T. M. S. Wolever, Glycaemic index methodology, Nutr. Res. Rev., 2005, 18, 145-145.

50 D. Bates, M. Mächler, E. Zurich, B. M. Bolker and S. C. Walker, Fitting linear mixed-effects models using lme4, J. Stat. Software, 2014, 67, 1-48.

51 N. Anders, K. Stott, P. R. Shewry, J. C. Mortimer, P. Dupree, T. Weimar, A. Lovegrove, R. A. C. Mitchell, J. Freeman, M. D. Wilkinson, T. Tryfona, M. Defoin-Platel, J. M. Baker and T. K. Pellny, Glycosyl transferases in family 61 mediate arabinofuranosyl transfer onto xylan in grasses, Proc. Natl. Acad. Sci. U. S. A., 2012, 109, 989-993.

52 H. N. Englyst, M. E. Quigley and G. J. Hudson, Determination of dietary fibre as non-starch polysaccharides with gas-liquid chromatographic, high-performance liquid chromatographic or spectrophotometric measurement of constituent sugars, Analyst, 1994, 119, 1497-1509.

53 H. Li, S. Dhital, A. J. Slade, W. Yu, R. G. Gilbert and M. J. Gidley, Altering starch branching enzymes in wheat generates high-amylose starch with novel molecular structure and functional properties, Food Hydrocolloids, 2019, 92, 51-59.

54 S. Dhital, F. J. Warren, P. J. Butterworth, P. R. Ellis and M. J. Gidley, Mechanisms of starch digestion by $\alpha$-amylase -Structural basis for kinetic properties, Crit. Rev. Food Sci. Nutr., 2017, 57, 875-892.

55 H. Patel, P. G. Royall, S. Gaisford, G. R. Williams, C. H. Edwards, F. J. Warren, B. M. Flanagan, P. R. Ellis and P. J. Butterworth, Structural and enzyme kinetic studies of retrograded starch: Inhibition of $\alpha$-amylase and consequences for intestinal digestion of starch, Carbohydr. Polym., 2017, 164, 154-161.

56 J. A. Delcour, C. Bruneel, L. J. Derde, S. V. Gomand, B. Pareyt, J. A. Putseys, E. Wilderjans and L. Lamberts, Fate of Starch in Food Processing: From Raw Materials to Final Food Products, Annu. Rev. Food Sci. Technol., 2010, 1, 87-111.

57 C. H. Edwards, F. J. Warren, G. M. Campbell, S. Gaisford, P. G. Royall, P. J. Butterworth and P. R. Ellis, A study of starch gelatinisation behaviour in hydrothermally-processed plant food tissues and implications for in vitro digestibility, Food Funct., 2015, 6, 3634-3641.

58 M. A. De Santis, O. Kosik, D. Passmore, Z. Flagella, P. R. Shewry and A. Lovegrove, Data set of enzyme fingerprinting of dietary fibre components (arabinoxylan and $\beta$-glucan) in old and modern Italian durum wheat genotypes, Data Brief, 2018, 16, 1062-1068.

59 C. M. Courtin and J. A. Delcour, Arabinoxylans and Endoxylanases in Wheat Flour Bread-making, J. Cereal Sci., 2002, 35, 225-243.

60 J. A. Delcour, X. Rouau, C. M. Courtin, K. Poutanen and R. Ranieri, Technologies for enhanced exploitation of the health-promoting potential of cereals, Trends Food Sci. Technol., 2012, 25, 78-86. 